\title{
Typical predictive eye movements during action observation without effector-specific motor simulation
}

\author{
Gilles Vannuscorps $^{1,2}$ - Alfonso Caramazza ${ }^{1,2}$
}

Published online: 21 December 2016

(C) Psychonomic Society, Inc. 2016

\begin{abstract}
When watching someone reaching to grasp an object, we typically gaze at the object before the agent's hand reaches it - that is, we make a "predictive eye movement" to the object. The received explanation is that predictive eye movements rely on a direct matching process, by which the observed action is mapped onto the motor representation of the same body movements in the observer's brain. In this article, we report evidence that calls for a reexamination of this account. We recorded the eye movements of an individual born without arms (D.C.) while he watched an actor reaching for one of two different-sized objects with a power grasp, a precision grasp, or a closed fist. D.C. showed typical predictive eye movements modulated by the actor's hand shape. This finding constitutes proof of concept that predictive eye movements during action observation can rely on visual and inferential processes, unaided by effector-specific motor simulation.
\end{abstract}

Keywords Action observation - Eye movements · Motor simulation $\cdot$ Single-case study

When watching someone grasping an object, we typically gaze at the object before the agent's hand reaches it - that is, we make a "predictive eye movement" to the object. What kind of representations and processes underlie these predictive eye movements?

Gilles Vannuscorps

gvannuscorps@ fas.harvard.edu

1 Department of Psychology, Harvard University, Cambridge, MA 02138, USA

2 Center for Mind/Brain Sciences, Università degli Studi di Trento, Mattarello 38122, Italy
Since the seminal work by Flanagan and Johansson (2003), the received explanation has been that predictive eye movements rely on a direct matching process by which the observed action is mapped onto the motor representation of the same body movements in the observer's brain. According to this account, this effector-specific motor simulation of the observed action would automatically activate, in the observer's brain, the predictive eye motor programs that accompany the execution of that action (Ambrosini, Costantini, \& Sinigaglia, 2011; Ambrosini et al., 2013; Ambrosini, Sinigaglia, \& Costantini, 2012; Costantini, Ambrosini, \& Sinigaglia, 2012a, 2012b; Elsner, D’Ausilio, Gredebäck, Falck-Ytter, \& Fadiga, 2013; Falck-Ytter, Gredebäck, \& von Hofsten, 2006). Two main sets of data are cited in support of this hypothesis. One is results showing that predictive eye movements during the observation of hand actions are influenced by the state of the motor system: They are delayed by a concurrent fingertapping task (Cannon \& Woodward, 2008), modulated by participants' hand position (Ambrosini et al., 2012), and hampered by the transient disruption of parts of the hand action production neural network (Costantini, Ambrosini, Cardellicchio, \& Sinigaglia, 2013; Elsner et al., 2013). The other is the finding of a relationship between infants' motor abilities and predictive eye movements (Ambrosini et al., 2013; Falck-Ytter et al., 2006).

However, these observations can also be accommodated by an alternative hypothesis, according to which predictive eye movements are driven mainly by visual and inferential mechanisms, but can be modulated by the state of the motor system (Eshuis, Coventry, \& Vulchanova, 2009; Falck-Ytter, 2012). There is indeed some evidence that motor activity can modulate the activity of visual brain areas thought to play a role in the perception of body shape and body movements (e.g., Astafiev et al., 2004). Thus, interfering with the motor system could have functional effects upon predictive eye movements 
because it disrupts normal recursive interactions between the motor and visual brain areas serving other purposes, such as action imitation (e.g., Buccino et al., 2004) or short-term memory (Moreau, 2013; Vannuscorps \& Caramazza, 2016). According to this hypothesis, the relationship between infants' motor abilities and predictive eye movements finds a natural explanation in the assumption that motor experience contributes not only to the learning of motor representations, but also to the development of spatiotemporal knowledge about the performed action, and that the latter, rather than the former, contributes to action anticipation (Southgate, 2013).

In this study, we used a novel approach to test whether predictive eye movements during action observation require a direct matching process. We reasoned that if eye movements during action observation require direct matching and effector-specific motor simulation, then predictive eye movements should be specific to actions that an observer can simulate within his or her own motor system. To test this prediction, we recorded the eye movements of an individual (D.C.) born without upper limbs (bilateral upper limb dysplasia) and no history of upper limb prosthetics or phantom limb sensations when watching an actor reaching for or grasping one of two target objects with a precision or a power grasp.

D.C.'s congenital lack of upper limbs has prevented him from learning the motor programs associated with the execution of the typical reaching and grasping actions shown in this study. Thus, unless one were to assume that motor programs of actions such as "grasping with the thumb and the index finger" are innate rather than learned by trial and error during development, we can conclude that D.C. does not have upper limb motor representations onto which the upper limb movements shown in this study could be mapped. Therefore, if predictive eye movements during action observation rely on direct matching and effector-specific motor simulation, D.C.'s eye movements when observing hand actions should be reactive, not predictive. If, however, predictive eye movements rely on visual and inferential mechanisms, then D.C.'s eye movements should be analogous to those of typically developed participants.

To ensure sensitivity in detecting possibly abnormal predictive eye movements in D.C., we measured control participants' eye movements when observing an actor reaching for one of two potential target objects (a small or a large one) with either a closed fist or a hand shape appropriate for grasping one of them (i.e., a precision or a power grasp). This experimental design provided three main advantages over designs involving only one target object: (1) Participants did not know the intention of the agent (which target and which hand shape) in advance, thereby ensuring that their predictive eye movements, if any, would be driven by the processing of cues inherent to the stimulus. (2) The design allowed for testing whether participants' eye movements were modulated by the presence of the actor's hand shape information, a modulation considered to be evidence for the crucial role of motor simulation in action perception (Ambrosini et al., 2011; Costantini et al., 2013; Falck-Ytter, 2012); (3) this design has been previously shown to be very sensitive in detecting even subtle impairments of predictive eye movements (Ambrosini et al., 2012; Costantini et al., 2013).

\section{Material and methods}

\section{Participant}

D.C. is a 53-year-old man whose performance in various experiments has already been reported (Vannuscorps, Pillon \& Andres, 2012; Vannuscorps, Andres \& Pillon, 2013, 2014). He has a master's degree in psychopedagogy. He has a congenital bilateral upper limb dysplasia due to in-utero thalidomide exposure. Congenital abnormalities include aplasia of the left upper limb (i.e., the most severe form of dysplasia, characterized by a complete absence of arm, forearm, hand, and fingers) and, on the right side, a shortened right arm ( $\pm 12 \mathrm{~cm}$ humerus or ulna) directly fused to a hand composed of Fingers 1 and 3 (shoulder, elbow, and wrist joints absent or not functional). D.C. can move his right upper extremity as a whole by a couple of centimeters in every direction and can hold light objects by squeezing them between his chest and shortened limb. However, the absence of most of the typical bones and joints in D.C.'s right upper extremity has prevented typical muscle insertion and development. Therefore, his finger mobility is too limited to allow him to make a precision or palm grip, and he cannot grasp or manipulate any object. Instead, D.C. developed fine motor skills of the feet from early childhood, which allow him to use his feet for many typically hand-related actions of daily life (e.g., writing with a pen, typewriting, eating with a fork, and washing himself). His physical and mental development was otherwise normal. D.C. never wore prosthetics and reports no history of phantom limb sensations or movements. D.C. has impaired vision of the left eye (visual acuity $=0.5 / 10$ ) due to an acquired eye injury, but normal vision of the right eye. Ten normally developed control participants also participated in the study (seven men, three women; participants S1 and S6 were left-handed, mean age $=30$ ). The study was approved by the biomedical ethic committee of the Cliniques Universitaires Saint-Luc, Brussels, and all participants gave their written informed consent prior to the study.

\section{Procedure and stimuli}

The stimuli, provided by Marcello Costantini (Ambrosini et al., 2011; Costantini et al., 2013), were video clips showing the right hand of an actor, viewed from a third-person perspective from the left side, moving forward $70 \mathrm{~cm}$ to reach one of two potential objects (a small or a large target object) 
positioned on a table $10 \mathrm{~cm}$ apart from each other, with either a closed fist ("no-shape" movement condition) or a hand shape ("shape" movement condition) appropriate for grasping them (either a precision or a power grasp). Four different object layouts were used to counterbalance the hand trajectories (see Ambrosini et al., 2011). The total number of different videos was thus $16(2$ Targets $\times 2$ Movement Conditions $\times 4$ Layouts). All videos had the same timing: They started with a 1,000-ms fixation cross superimposed on the actor's hand (fixation phase), then the actor's hand moved toward one of the two target objects for $1,000 \mathrm{~ms}$, and then the last frame of the video was shown for $500 \mathrm{~ms}$.

During the experiment, participants were seated with the head stabilized by a head-and-chin rest at $52 \mathrm{~cm}$ from a $17 \mathrm{in}$. computer screen $(395 \times 290 \mathrm{~mm}, 1,280 \times 1,024$ pixels, $60 \mathrm{~Hz}$ ). In that way, all the videos were sized $21.4^{\circ} \times 16.16^{\circ}$ of visual angle $(197.5 \times 136 \mathrm{~mm})$. Every trial started with the presentation of a central white fixation cross lasting 500, 1,000 , or $1,500 \mathrm{~ms}$ (balanced across conditions) that the participants were asked to fixate. Then the video was displayed, and participants were asked to fixate the white cross superimposed on the actor's hand (fixation phase) and to "simply watch the video" (Ambrosini et al., 2011). Participants performed two blocks of 48 trials, preceded by a familiarization phase and ten practice trials. Both blocks were preceded by standard 9-point calibration and validation of the participant's eye-gaze position. In each block, the four layouts - two movement conditions and two target objectswere mixed in a different random order.

The presentation of the stimuli was controlled by the EPrime software (Psychology Software Tools, Pittsburgh, PA), and participants' eye movements were recorded by an EyeLink 1000 desktop-mounted eyetracker (SR Research, Canada; sampling rate of $1000 \mathrm{~Hz}$, average accuracy range $0.25^{\circ}-0.5^{\circ}$, gaze tracking range of $32^{\circ}$ horizontally and $25^{\circ}$ vertically).

\section{Results}

During the recording session, gaze traces were parsed by means of a saccadic velocity-threshold identification algorithm implemented in EyeLink 1000. EyeLink Data Viewer (SR Research, Canada) was used to extract the timing and position of participants' fixations in each trial. To compensate for drift in the eyetracker signal's offset over time, a corrective adjustment was applied to the position of participants' fixations, based on the difference between the raw position of the eye at the beginning of each trial and the position of the fixation cross participants were asked to fixate (Hornof \& Halverson, 2002).

Regions of interest (ROIs) centered on the actor's hand (hand ROI) and the target object (target ROI) were created for the 16 different video clips. The ROIs were circles $0.2^{\circ}$ larger than the object, to compensate for noise in the eyetracking (Costantini et al., 2013; Costantini et al., 2012a). Then, for each trial we calculated the time of the first saccade from the hand ROI (gaze-onset time) and the time of the first fixation on the target ROI (gaze arrival time), relative to the hand movement onset and offset (Ambrosini et al., 2011).

These analyses were performed over two dependent variables: an "accuracy" measure, corresponding to the percentage of trials on which participants' gazes fixated on the target ROI before the hand movement offset (Ambrosini et al., 2011), and a "latency" measure, computed for each participant by subtracting the mean gaze arrival time from the hand movement offset time (thus, the larger the latency, the larger the anticipation). Trials in which participants did not fixate within the hand ROI at hand movement onset (end of the fixation phase) were discarded from the accuracy analyses ( $2 \%$ and $5 \%$ of trials for D.C. and controls, respectively). For the latency analysis, we further discarded trials in which participants did not fixate the target ROI before the end of the trial (3\% and $38 \%$ of trials for D.C. and controls, respectively) and trials in which the gaze arrival times deviated from the participant's mean by two $S D$ s in each movement condition (3.5\% and 3.9\% of trials for D.C. and controls, respectively).

Distinct analyses were performed to test whether D.C.'s eye movements when observing hand actions were "predictive" (i.e., over the whole set of stimuli) and whether the hand actions were influenced by the availability of information about the actor's hand shape (i.e., we contrasted the stimuli with and without hand shape information).

\section{General analyses of D.C.'s and controls' eye movements}

The direct-matching hypothesis predicts that D.C.'s eye movements when observing hand actions should be reactive, not predictive. Contrary to this expectation, we found that D.C.'s eye movements were predictive: He fixated on the target ROI before the hand offset on $82 \%$ of trials and, on average, fixated the target ROI $291 \mathrm{~ms}$ before the hand offset (Fig. 1). In fact, D.C. had a nonsignificant tendency [both modified $t$ tests, $t \mathrm{~s}(9)$ $<2, p \mathrm{~s}>.05$; Crawford \& Howell, 1998] to fixate on the target ROI before the hand offset more often ( $82 \%$ of the trials) and earlier (mean anticipation: $291 \mathrm{~ms}$ ) than the controls (48\% and $179 \mathrm{~ms})$. To further investigate this result, we then used a onesided Bayesian $t$ test (with a default Cauchy prior width of $r=$ .707 for effect size on the alternative hypothesis, as specified by Rouder, Morey, Speckman, \& Province, 2012), with items as the random variable, to quantify the evidence in the data for the hypotheses that D.C. anticipated the outcome of the agent's action (1) less accurately or (2) less rapidly than the controls (H1), and for the corresponding null hypotheses (H0). These analyses, performed with Morey and Rouder's (2015) BayesFactor R package, yielded two Bayes factors $(\mathrm{H} 1 / \mathrm{H} 0)$ of 0.06 , indicating that the observed data were 15 times more 
a

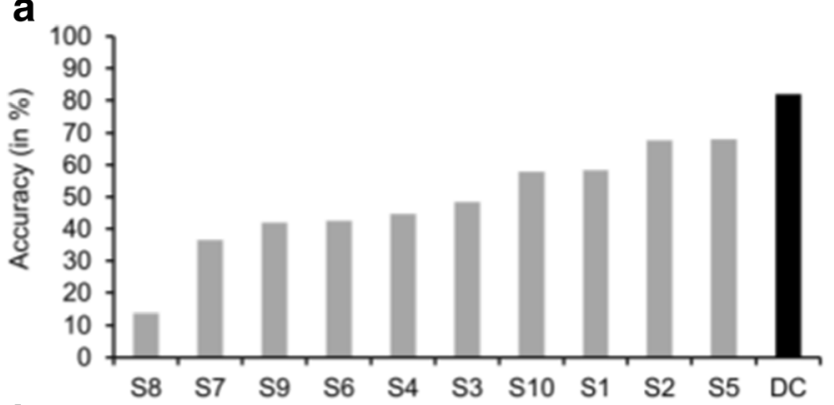

b

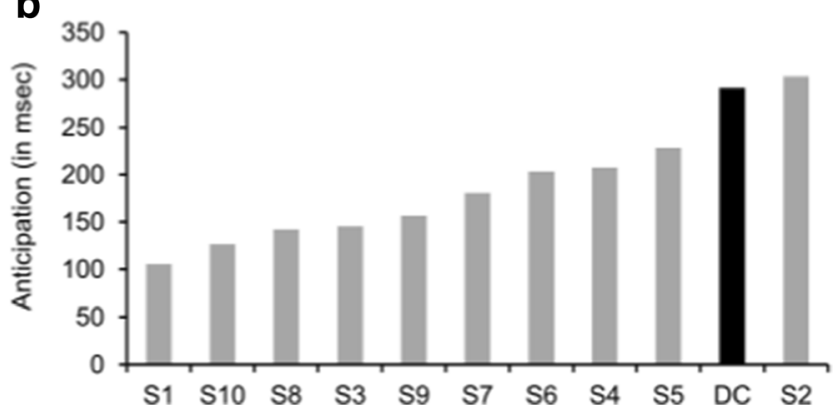

Fig. 1 Accuracies and mean anticipation times in D.C. and the control participants, displayed in ascending order as a function of the participants' anticipation. (A) Accuracy: Percentages of trials in which participants fixated the target region of interest (ROI) before the hand movement offset. (B) Anticipation: Participants' mean eye arrival times on the target ROI, relative to the hand movement offset. black, D.C.; gray, control participants

likely under the null hypothesis than under the alternative hypothesis. According to Jeffreys (1961), this constitutes strong evidence in favor of the null hypothesis.

\section{Effect of the availability of information about the actor's hand shape}

The effect of hand shape information on participants' eye movements is considered to be evidence for the crucial role of motor simulation in action perception. Our results, shown in Fig. 2, call for a reexamination of this claim: D.C. was more accurate $($ shape $=93.62 \%$, no-shape $=70.21 \%), \chi^{2}(1)=8.69$, $p=.003$, and made earlier fixations on the target (shape $=-$ $344 \mathrm{~ms}$, no-shape $=-231 \mathrm{~ms}$ ) when information about the actor's hand shape was available, and the influence of the presence of the actor's hand shape was larger in D.C. than in the control group, in terms of both accuracy and latency (Bayesian standardized difference test, both $p \mathrm{~s}<.05$; Crawford \& Garthwaite, 2007).

We further investigated this difference between D.C. and the control participants. A reliably larger influence of the presence of the actor's hand shape information in D.C. would indicate that engaging in motor simulation is not only not necessary but could interfere with predictive eye movements. To that end, we first conducted Bayesian analyses of variance (ANOVAs) on participants' accuracies and latencies, with items as the random variable, to quantify the evidence in the

\section{a}

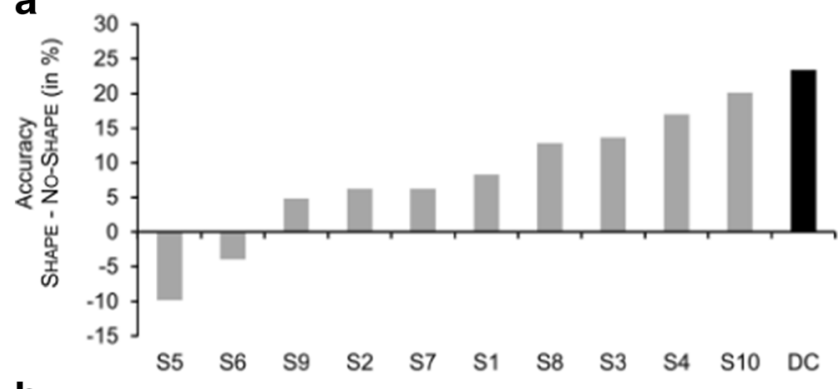

b

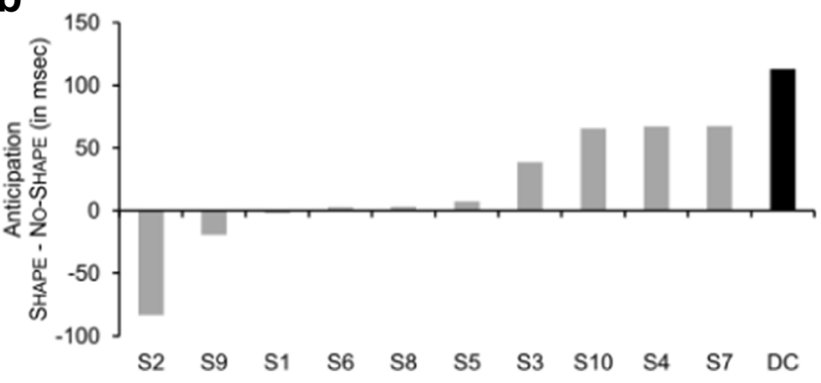

Fig. 2 Differences in accuracy and anticipation times between the shape and no-shape movement conditions for D.C. and each control participant, in ascending order as a function of the size of the influence of the shape information. (A) Differences in percentages of trials in which participants fixated the target ROI before the hand movement offset, between the shape and no-shape conditions. (B) Differences in each participant's mean eye arrival times on the target ROI relative to the hand movement offset, between the shape and no-shape conditions. black, D.C.; gray, control participants

data for the hypothesis that there was an interaction between group (D.C. vs. controls) and stimuli (shape vs. no-shape) and the corresponding null hypothesis, according to which there was no interaction (Rouder et al., 2012). Comparison of the Bayes factors between the ANOVA model including only the main effects (H0) and the ANOVA model also including their interaction (H1, with a default Cauchy prior width of $r=.707$ for effect size), performed with the BayesFactor R package (Morey \& Rouder, 2015), provided only anecdotal support for the interaction model in the latency analysis $(1: 1.4)$ and for the absence of an interaction in the accuracy analysis (1.5:1).

Then, we also looked at the data of an independent group of 15 typically developed healthy participants reported by Ambrosini et al. (2011; see Table 1). This group of participants tended to be more influenced by the presence of an actor's hand shape that were the control participants tested in this study (see Table 1). Because these participants were tested with the same stimuli and procedures as the participants tested in our study, this difference is likely the result of the large interindividual differences in predictive eye movements that were already visible among our control participants (Figs. 1 and 2). Importantly, however, the influence of the presence of the actor's hand shape in this independent group of 15 typically developed participants was very similar to that found in D.C. (see Table 1). These two additional analyses suggest that D.C.'s predictive eye movements were very similar to those of control participants. 
Table 1 Accuracies and mean anticipation times for the whole set of stimuli and for the stimuli of the no-shape and shape movement conditions, along with the differences between conditions, for D.C., the control participants, and 15 typically developed healthy participants reported by Ambrosini et al. (2011)

\begin{tabular}{lllll}
\hline & & D.C. & Control Participants & $\begin{array}{c}\text { Control Participants From } \\
\text { Ambrosini et al. (2011) }\end{array}$ \\
\hline Accuracy & All stimuli & $82 \%$ & $48 \%$ & $73.2 \%$ \\
& Shape & $93.6 \%$ & $51.75 \%$ & $82 \%$ \\
& No-shape & $70.2 \%$ & $44.19 \%$ & $65 \%$ \\
Anticipation & Difference & $23.4 \%$ & $7.56 \%$ & $17 \%$ \\
& All stimuli & $-291 \mathrm{~ms}$ & $-179 \mathrm{~ms}$ & n.a. \\
& Shape & $-344 \mathrm{~ms}$ & $-186 \mathrm{~ms}$ & $-143 \mathrm{~ms}$ \\
& No-shape & $-231 \mathrm{~ms}$ & $-171 \mathrm{~ms}$ & $+10 \mathrm{~ms}$ \\
& Difference & $113 \mathrm{~ms}$ & $15 \mathrm{~ms}$ & $153 \mathrm{~ms}$ \\
\hline
\end{tabular}

\section{Discussion and conclusion}

We found that when observing an actor reaching to grasp an object, a person born without upper limbs (D.C.), who therefore had no effector-specific motor representation of the observed action, gazed at the target object before the hand movement offset as often and quickly as typically developed control participants. We also found that, like the control participants, D.C. showed higher accuracy and earlier saccadic movements when he observed a grasping hand than when he observed a reaching hand devoid of a grasping shape. This modulation of predictive eye movements by the actor's hand shape had previously been considered an index of the crucial role of motor simulation in predictive eye movements during action observation (Ambrosini et al., 2011; Costantini et al., 2013; Falck-Ytter, 2012).

These findings cannot easily be dismissed by appeals to a lack of power or sensitivity. We used a design that has previously been shown to be very sensitive in detecting even subtle impairments of predictive eye movements (Ambrosini et al., 2012; Costantini et al., 2013), and, if anything, D.C. had a tendency to anticipate more quickly and more often, and to be more influenced by the presence of the actor's hand shape, than were typically developed control participants. Furthermore, the Bayes factors, which unlike traditional $p$ values allow experimenters to quantify the evidence for the null hypothesis, provided strong support in favor of the hypothesis that D.C.'s eye movements were not less predictive or less influenced by the actor's hand shape than the controls' eye movements.

Our findings thus constitute proof that it is possible to account for typical predictive eye movements, and to explain the modulation of eye movements by actor's hand shape information, without appealing to the concept of effector-specific motor simulation. As a corollary, our findings suggest that typical predictive eye movements during action observation can rely exclusively on visual and inferential processes, unaided by motor simulation (Eshuis et al., 2009). According to this hypothesis, when an action is perceived, a visuo-perceptual analysis of the observed scene (i.e., of the actor's body shape and motion, of objects in the scene, and of context) provides a visual description of the action, which serves as input to an inferential system that, to predict the most likely outcome of the observed action (e.g., Eshuis et al., 2009), makes use of a variety of information and knowledge ("internal models") about the actor, the environment, and the observed action that have been accumulated over previous experience.

An alternative interpretation of our findings is that predictive eye movements during action observation rely on a kind of motor simulation that is not effector-specific. According to this account, D.C.'s eye movements would be driven by his covertly imitating the observed hand movements with his lower limbs. However, the very different skeletal and muscular features and degrees of freedom of the hands and feet and fingers and toes make it virtually impossible to imitate the hand movements used in our experiment with the feet. The feet allow for neither grasping a large object with a palm power grip, nor grasping a small object with a precision grip opposing two fingertips. It is unclear, therefore, how a mechanism of direct, observation-execution matching could underlie D.C.'s predictive eye movements if he has never executed the observed motor act-for instance, grasping a large object with a palm power grip.

In conclusion, our findings challenge the currently received explanation of predictive eye movements during action observation, which holds that they cannot be achieved by visual and inferential processes alone, but require a direct mapping process of the observed action onto the motor representation of the same body movements in the observer's brain. Of course, the extent to which these results will generalize to typically developed participants and to other types of stimuli (e.g., degraded stimuli or stimuli in first person perspective) remains an open question. It is possible, for instance, that predictive 
eye movements could rely on visual and inferential computations in D.C., but require motor simulation in typically developed participants. However, as we discussed in the introduction, currently no compelling evidence supports this view. In this context, our finding underscores at the very least the need for a shift in the burden of proof relative to the question of the role of motor processes in predictive eye movements during action observation.

Author note We are grateful to Marcello Costantini, who provided the experimental material, and to Angelika Lingnau, for her help with the eyetracker. This research was supported by the Fondazione Cassa di Risparmio di Trento e Rovereto and by the Provincia Autonoma di Trento.

\section{References}

Ambrosini, E., Costantini, M., \& Sinigaglia, C. (2011). Grasping with the eyes. Journal of Neurophysiology, 106, 1437-1442.

Ambrosini, E., Reddy, V., de Looper, A., Costantini, M., Lopez, B., \& Sinigaglia, C. (2013). Looking ahead: anticipatory gaze and motor ability in infancy. PLOS ONE, 8, e67916. doi:10.1371/journal. pone. 0067916

Ambrosini, E., Sinigaglia, C., \& Costantini, M. (2012). Tie my hands, tie my eyes. Journal of Experimental Psychology, 38, 263-266.

Astafiev, S. V., Stanley, C. M., Shulman, G. L., \& Corbetta, M. (2004). Extrastriate body area in human occipital cortex responds to the performance of motor actions. Nature Neuroscience, 7, 542-548.

Buccino, G., Vogt, S., Ritzi, A., Fink, G. R., Zilles, K., Freund, H.-J., \& Rizzolatti, G. (2004). Neural circuits underlying imitation learning of hand actions: An event-related fMRI study. Neuron, 42, 323-334.

Cannon, E. N., \& Woodward, A. L. (2008). Action anticipation and interference: A test of prospective gaze. In B. C. Love, K. McRae, \& V. M. Sloutsky (Eds.), Proceedings of the 30th Annual Conference of the Cognitive Science Society (pp. 981-984). Austin, TX: Cognitive Science Society.

Costantini, M., Ambrosini, E., Cardellicchio, P., \& Sinigaglia, C. (2013). How your hand drives my eyes. Social Cognitive and Affective Neuroscience, 9, 705-711.

Costantini, M., Ambrosini, E., \& Sinigaglia, C. (2012a). Does how I look at what you're doing depend on what I'm doing? Acta Psychologica, 141, 199-204. doi:10.1016/j.actpsy.2012.07.012

Costantini, M., Ambrosini, E., \& Sinigaglia, C. (2012b). Out of your hand's reach, out of my eyes' reach. Quarterly Journal of Experimental Psychology, 65, 848-855. doi:10.1080/17470218.2012.679945
Crawford, J. R., \& Garthwaite, P. H. (2007). Comparison of a single case to a control or normative sample in neuropsychology: Development of a Bayesian approach. Cognitive Neuropsychology, 24, 343-372.

Crawford, J. R., \& Howell, D. C. (1998). Comparing an individual's test score against norms derived from small samples. Clinical Neuropsychologist, 12, 482-486.

Elsner, C., D’Ausilio, A., Gredeback, G., Falck-Ytter, T., \& Fadiga, L. (2013). The motor cortex is causally related to predictive eye movements during action observation. Neuropsychologia, 51, 488-492.

Eshuis, R., Coventry, K. R., \& Vulchanova, M. (2009). Predictive eye movements are driven by goals, not by the mirror neuron system. Psychological Science, 20, 438-440.

Falck-Ytter, T. (2012). Predicting other people's action goals with low-level motor information. Journal of Neurophysiology, 107, 2923-2925.

Falck-Ytter, T., Gredebäck, G., \& von Hofsten, C. (2006). Infants predict other people's action goals. Nature Neuroscience, 9, 878-879.

Flanagan, J. R., \& Johansson, R. S. (2003). Action plans used in action observation. Nature, 424, 769-771.

Hornof, A. J., \& Halverson, T. (2002). Cleaning up systematic error in eye-tracking data by using required fixation locations. Behavior Research Methods, Instruments, \& Computers, 34, 592-604. doi:10.3758/BF03195487

Jeffreys, H. (1961). Theory of probability (3rd ed.). Oxford, UK: Oxford University Press.

Moreau, D. (2013). Motor expertise modulates movement processing in working memory. Acta Psychologica, 142, 356-361.

Morey, R. D., \& Rouder, J. N. (2015). BayesFactor: Computation of Bayes factors for common designs ( $\mathrm{R}$ package version 0.9.10-1). Retrieved from http://CRAN.R-project.org/package=BayesFactor

Rouder, J. N., Morey, R. D., Speckman, P. L., \& Province, J. M. (2012). Default Bayes factors for ANOVA designs. Journal of Mathematical Psychology, 56, 356-374. doi:10.1016/j. jmp.2012.08.001

Southgate, V. (2013). Do infants provide evidence that the mirror system is involved in action understanding? Consciousness and Cognition, 22, 1114-1121.

Vannuscorps, G., Andres, M., \& Pillon, A. (2013). When does action comprehension need motor involvement? Evidence from upper limb dysplasia. Cognitive Neuropsychology, 30, 253-283.

Vannuscorps, G., Andres, M., \& Pillon, A. (2014). Is motor knowledge part and parcel of the concept of manipulable artifacts ? Clues from a case of upper limb aplasia. Brain and Cognition, 84, 132-140.

Vannuscorps, G., \& Caramazza, A. (2016). Impaired short-term memory for hand postures in individuals born without hands. Cortex, 83, $136-138$.

Vannuscorps, G., Pillon, A., \& Andres, M. (2012). The effect of biomechanical constraints in the hand laterality judgment task: Where does it come from? Frontiers in Human Neuroscience, 6, 299. doi:10.3389/fnhum.2012.00299 